\title{
Research Article \\ Creep Modeling in a Composite Rotating Disc with Thickness Variation in Presence of Residual Stress
}

\author{
Vandana Gupta and S. B. Singh \\ Department of Mathematics, Punjabi University, Punjab Patiala 147 002, India \\ Correspondence should be addressed to Vandana Gupta, vaggarwal2584@gmail.com
}

Received 30 March 2012; Revised 4 August 2012; Accepted 5 September 2012

Academic Editor: Frank Werner

Copyright (C) 2012 V. Gupta and S. B. Singh. This is an open access article distributed under the Creative Commons Attribution License, which permits unrestricted use, distribution, and reproduction in any medium, provided the original work is properly cited.

Steady-state creep response in a rotating disc made of Al-SiC (particle) composite having linearly varying thickness has been carried out using isotropic/anisotropic Hoffman yield criterion and results are compared with those using von Mises yield criterion/Hill's criterion ignoring difference in yield stresses. The steady-state creep behavior has been described by Sherby's creep law. The material parameters characterizing difference in yield stresses have been used from the available experimental results in literature. Stress and strain rate distributions developed due to rotation have been calculated. It is concluded that the stress and strain distributions got affected from the thermal residual stress in an isotropic/anisotropic rotating disc, although the effect of residual stress on creep behavior in an anisotropic rotating disc is observed to be lower than those observed in an isotropic disc. Thus, the presence of residual stress in composite rotating disc with varying thickness needs attention for designing a disc.

\section{Introduction}

Residual stress significantly affects the engineering properties of materials and structural components, notably fatigue life, distortion, dimensional, corrosion resistance, brittle fracture, and so forth. For that reason, the residual stress analysis is an important stage in the design of parts and structural elements. The thermal residual stresses induced due to thermal mismatch between the metal matrix and the ceramic reinforcement in metal matrix composite may impart plastic deformation to the matrix. Thermal mismatch strains also may quite often crack the matrix resulting in a relaxation of the residual stresses. Presence of thermal residual stresses can induce the asymmetry in the tensile and compressive yield stresses of the composite. Residual stresses may be reduced or eliminated by annealing, by plastic deformation, or just by letting the piece at room temperature enough time. Because of its influence on the properties, the residual stress in composites has been the subject of several studies, both experimentally and analytically. Bhatnagar et al. [1] have performed 
steady-state creep analysis of orthotropic rotating discs having constant thickness, linearly varying thickness, and hyperbolically varying thickness. They have used Norton's power law to describe creep behavior of the disc material and concluded that by selecting an optimum profile for the disc, the stress and strain rate in the disc may be reduced. Arsenault and Taya [2] investigated the magnitude of the thermal residual stresses by determining the difference of the yield stresses between tension and compression resulting from the thermal residual stresses. Mishra and Pandey [3] have claimed that Sherby's constitutive creep model works better than Norton's creep law to describe the creep behavior of aluminum matrix composites. Pandey et al. [4] have studied the steady-state creep behavior of Al$\mathrm{SiCp}$ composites under uniaxial loading condition in the temperature range between $623 \mathrm{~K}$ and $723 \mathrm{~K}$ for different combinations of particle sizes $(1.7 \mu \mathrm{m}, 14.5 \mu \mathrm{m}$, and $45.9 \mu \mathrm{m})$ and with varying particle content $(10 \mathrm{vol} \%, 20 \mathrm{vol} \%$, and $30 \mathrm{vol} \%)$ and found that the composite with finer particle size has better creep resistance than that containing coarser ones. Davis and Allison [5] investigated that the mismatch in the coefficient of thermal expansion between the $\mathrm{SiC}$ particle and the aluminum alloy metal matrix gave rise to a high density of dislocation both at and near the reinforcement/matrix interface. The enhanced expansion of the matrix induced plastic deformation during cooling with an associated increase in the density of dislocations. Hu and Huang [6] proposed an analytical method to predict the influence of residual stress on the elastic-plastic behavior of a general composite. The model incorporates the microstructural parameters like fiber shape, orientation, distribution, and volume fraction. The computed results show that the influence of residual stress on the macroscopic properties depends closely on the microstructures of the composite. Jahed and Shirazi [7] investigated loading and residual stresses and associated strains and displacements in thermoplastic rotating discs at elevated temperatures. Orcan and Eraslan [8] investigated the distribution of stress, displacement, and plastic strain in a rotating elastic-plastic solid disc of variable thickness in a power function. The analysis is based on Tresca's yield condition. By employing a variable thickness disc the plastic limit angular velocity increases and the magnitude of stresses and deformations in the disc reduces. Singh and Ray [9] proposed a new yield criterion for residual stress, which at appropriate limits reduces to Hill anisotropic and Hoffman anisotropic yield criterion, and carried out analysis of steady-state creep in a rotating disc made of $\mathrm{Al}-\mathrm{SiCw}$ composite using this criterion and compared the results obtained using Hill anisotropic yield criterion ignoring difference in yield stresses. Singh and Ray [10] studied the effect of thermal residual stress on the steadystate creep behavior of a rotating disc made of composite using isotropic Hoffman yield criterion while describing the creep by Norton's power law. They concluded that the tensile residual stress significantly affects the strain rates in the disc when compared with the strain rate in the disc without residual stress. Gupta et al. [11] have analyzed steady-state creep in isotropic aluminum silicon carbide particulate rotating disc. The creep behavior has been described by Sherby's law. The authors concluded that the tangential as well as radial stress distribution in the disc does not vary significantly for various combinations of material parameters and operating temperatures. Moreover the tangential as well as radial strain rates in the disc reduce significantly with reducing particle size, increasing particle content, and decreasing operating temperature. Jahed et al. [12] observed that the use of variable thickness disc helps in minimizing the weight of disc in aerospace applications. There are numerous applications for gas turbine discs in the aerospace industry such as in turbojet engines. These discs normally work under high temperatures while subjected to high angular velocities. Sayman [13] investigated elastic-plastic and residual stresses in thermoplastic composite laminated plates under linear thermal loading and also carried 
out an elastic-plastic, thermal stress analyses on thermoplastic composite disc reinforced with steel fibers under uniform temperature distribution and concluded that plastic yielding expands both around inner and outer surfaces and that plastic flow is highest at the inner surface. Singh [14] has performed creep analysis in an anisotropic composite disc rotating at $15,000 \mathrm{rpm}$ and undergoing steady-state creep at $561 \mathrm{~K}$ following Norton's power law. The presence of anisotropy leads to significant reduction in the tangential and radial strain rates over the entire disc and helps in restraining creep response both in the tangential and in the radial directions. Xuan et al. [15] have studied the time-dependent deformation and fracture performance of multimaterial system and structures at elevated temperature. He developed a microregion deformation measuring technique which allows the direct measurement of the full creep strain fields of multimaterial system at higher temperature. Chamoli et al. [16] have studied the effect of anisotropy on the stress and strain rates and concluded the anisotropy of the material has a significant effect on the creep of a rotating disc. The creep behavior is described by Sherby's law. Singh and Rattan [17] have investigated the stress distributions and the resulting creep deformation in isotropic rotating disc having constant thickness and made of silicon carbide particulate reinforced aluminium base composite in presence of thermal residual stress. It is concluded that the presence of the tensile residual stress affects the distribution of stresses and strain in the disc with constant thickness. Chen et al. [18] have investigated the effects of material gradients on the creep stress and strain of a pressurized tank, which is assumed to be made of functionally graded materials. They have concluded that the magnitude of the creep strain is influenced by the elastic modulus distribution as well as the creep property distribution inside the functionally graded materials and achieved some fundamental knowledge of the materials distribution to reduce the maximum creep stress/strain level inside the functionally graded materials tank. Gupta and Singh [19] have studied the effect of anisotropy on the stress and strain rates in composite disc made of anisotropic material (6061Al-30\% vol $\mathrm{SiC}_{p}$ ) and concluded that the anisotropy of the material has a significant effect on the creep of a rotating disc with varying thickness.

In this paper, the steady-state creep has been investigated for composite rotating disc made of material 6061Al base alloy containing $20 \mathrm{vol} \%$ of $\mathrm{SiC}$ (particle). The analysis has been done with/without thermal residual stresses for isotropic/anisotropic disc of linearly varying thickness. The creep behavior has been described by Sherby's constitutive model.

\section{Mathematical Formulation}

Consider a thin orthotropic composite disc of $6061 \mathrm{Al}^{-\mathrm{SiC}_{p}}$ of density $\rho$ and rotating at a constant angular speed $\omega$ radian/sec. The thickness of the disc is assumed to be $h$ and $a$ and $b$ be inner and outer radii of the disc, respectively. Let $I$ and $I_{0}$ be the moment of inertia of the disc at inner radius $a$ and outer radius $r$ and $b$, respectively. $A$ and $A_{0}$ are the area of cross-section of disc at inner radius $a$ and outer radius $r$ and $b$, respectively. Then

$$
\begin{gathered}
I=\int_{a}^{r} h r^{2} d r, \quad I_{0}=\int_{a}^{b} h r^{2} d r, \\
A=\int_{a}^{r} h d r, \quad A_{0}=\int_{a}^{b} h d r, \\
\sigma_{\theta_{\text {avg }}}=\frac{1}{A_{0}} \int_{a}^{b} h \sigma_{\theta} d r .
\end{gathered}
$$


For the purpose of analysis of the disc the following assumptions are made.

(1) Material of disc is orthotropic and incompressible.

(2) Elastic deformations are small for the disc and therefore they can be neglected as compared to creep deformation.

(3) Axial stress in the disc may be assumed to be zero as thickness of disc is assumed to be very small compared to its diameter.

(4) The composite shows a steady-state creep behavior, which may be described by following Sherby's law [20]:

$$
\dot{\bar{\varepsilon}}=\left(M\left(\bar{\sigma}-\sigma_{0}\right)\right)^{n},
$$

where $M=E^{-1}\left(A D_{\lambda} \lambda^{3} /\left|b_{r}\right|^{5}\right)^{1 / n}$, where $\dot{\bar{\varepsilon}}, \bar{\sigma}, n, \sigma_{0}, A, D_{\lambda}, \lambda, b, E$ be the effective strain rate, effective stress, the stress exponent, threshold stress, a constant, lattice diffusivity, the subgrain size, the magnitude of burgers vector, Young's modulus. The values of creep parameters $m$ and $\sigma_{0}$ are described by the following regression equations as a function of the particle size $(P)$ and the percentage of dispersed particles $(V)$ apart from the temperature $(T)$, which extracted from the available experimental results of Panday et al. [4]:

$$
\begin{gathered}
m=e^{-35.38} P^{0.2077} T^{4.98} V^{-0.622}, \\
\sigma_{0}=-0.03507 P+0.01057 T+1.00536 V-2.11916 .
\end{gathered}
$$

The different material combinations in the composite are conceptually replaced by an equivalent monolithic material that has the yielding and creep behavior similar to those displayed by the composite. Taking reference frame along the principal directions of $r, \theta$ and $z$, the generalized constitutive equations for an anisotropic disc under multiaxial stress condition are given as

$$
\begin{aligned}
& \dot{\varepsilon}_{r}=\frac{\dot{\bar{\varepsilon}}}{2 \bar{\sigma}}\left\{(G+H) \sigma_{r}-H \sigma_{\theta}-G \sigma_{z}+\left(f_{c}-f_{t}\right)\right\}, \\
& \dot{\varepsilon}_{\theta}=\frac{\dot{\bar{\varepsilon}}}{2 \bar{\sigma}}\left\{(H+F) \sigma_{\theta}-F \sigma_{z}-H \sigma_{r}+\left(f_{c}-f_{t}\right)\right\}, \\
& \dot{\varepsilon}_{z}=\frac{\dot{\bar{\varepsilon}}}{2 \bar{\sigma}}\left\{(F+G) \sigma_{z}-G \sigma_{r}-F \sigma_{\theta}+\left(f_{c}-f_{t}\right)\right\},
\end{aligned}
$$

where the effective stress, $\bar{\sigma}$, is given by

$$
\bar{\sigma}=\left\{\frac{1}{(G+H)}\left[F\left(\sigma_{\theta}-\sigma_{z}\right)^{2}+G\left(\sigma_{z}-\sigma_{r}\right)^{2}+H\left(\sigma_{r}-\sigma_{\theta}\right)^{2}\right]\right\}^{1 / 2}
$$

where $F, G$, and $H$ are anisotropic constants of the material. $\dot{\varepsilon}_{r}, \dot{\varepsilon}_{\theta}, \dot{\varepsilon}_{z}$ and $\sigma_{r}, \sigma_{\theta}, \sigma_{z}$ are the strain rates and the stresses, respectively, in the direction $r, \theta$ and $z . \dot{\bar{\varepsilon}}$ is the effective strain 
rate, $\bar{\sigma}$ is the effective stress, and $f_{c}, f_{t}$ are uniaxial compression and tensile yield stresses, respectively. For biaxial state of stress $\left(\sigma_{r}, \sigma_{\theta}\right)$, the effective stress is

$$
\bar{\sigma}=\left\{\frac{1}{(G+H)}\left\{F \sigma_{\theta}^{2}+G \sigma_{r}^{2}+H\left(\sigma_{r}-\sigma_{\theta}\right)^{2}\right\}\right\}^{1 / 2} .
$$

Using (2.5) and (2.9), (2.5) can be rewritten as

$$
\dot{\varepsilon}_{r}=\frac{d \dot{u}_{r}}{d r}=\frac{\sqrt{F(G+H)}\left[((G / F)+(H / F)) x-(H / F)+\left(\left(f_{c}-f_{t}\right) / \sigma_{\theta}\right)\right]\left[M\left(\bar{\sigma}-\sigma_{0}\right)\right]^{n}}{2\left[((G / F)+(H / F)) x^{2}-2(H / F) x+((G / F)+(H / F))\right]^{1 / 2}} .
$$

Similarly from (2.6)

$$
\dot{\varepsilon}_{\theta}=\frac{\dot{u}_{r}}{r}=\frac{\sqrt{F(G+H)}\left[(1+(H / F))-(H / F) x+\left(\left(f_{c}-f_{t}\right) / \sigma_{\theta}\right)\right]\left[M\left(\bar{\sigma}-\sigma_{0}\right)\right]^{n}}{2\left[((G / F)+(H / F)) x^{2}-2(H / F) x+(1+(H / F))\right]^{1 / 2}} .
$$

From the material's incompressibility assumption, it follows that

$$
\dot{\varepsilon}_{z}=-\left(\dot{\varepsilon}_{r}+\dot{\varepsilon}_{\theta}\right)
$$

where $x(r)=\sigma_{r} / \sigma_{\theta}$ is the ratio of radial and tangential stresses and $\dot{u}_{r}=d u / d t$ is the radial deformation rate.

Dividing (2.10) by (2.11)

$$
\phi(r)=\frac{((G / F)+(H / F)) x-(H / F)+\left(\left(f_{c}-f_{t}\right) / \sigma_{\theta}\right)}{(1+(H / F))-(H / F) x+\left(\left(f_{c}-f_{t}\right) / \sigma_{\theta}\right)}
$$

where

$$
\phi(r)=\frac{d \dot{u}_{r}}{d r} \cdot \frac{\dot{u}_{r}}{r} \Longrightarrow \frac{d \dot{u}_{r}}{\dot{u}_{r}}=\frac{\phi(r)}{r} d r
$$

Integrating and taking limit $a$ to $r$ on both sides

$$
\dot{u}_{r}=\dot{u}_{r_{i}} \exp \int_{a}^{r} \frac{\phi(r)}{r} d r
$$

where $\dot{u}_{r_{i}}$ is the radial deformation rate at the inner radius. Dividing (2.15) by $r$ and equated to $(2.11)$

$$
\bar{\sigma}-\sigma_{0}=\frac{\left(\dot{u}_{r_{i}}\right)^{1 / n}}{M} \psi(r)
$$


where

$$
\psi(r)=\left\{\frac{2}{r} \cdot \frac{\left[((H / F)+(G / F)) x^{2}-(2 H x / F)+(1+(H / F))\right]^{1 / 2}}{\sqrt{F(G+H)}\left[(1+(H / F))-(H / F) x+\left(\left(f_{c}-f_{t}\right) / \sigma_{\theta}\right)\right]} \exp \cdot \int_{a}^{r} \frac{\phi(r) d r}{r}\right\}^{1 / n} .
$$

Substituting $\bar{\sigma}$ from (2.9) to (2.16), it gives

$$
\begin{aligned}
& \left\{(F /(G+F))\left[((G / F)+(H / F)) x^{2}-2(H / F) x+((H / F)+1)\right]\right\}^{1 / 2} \sigma_{\theta}-\sigma_{0}=\frac{\left(\dot{u}_{r_{i}}\right)^{1 / n}}{M} \psi(r) \\
& \Longrightarrow \sigma_{\theta}=\frac{\left(\dot{u}_{r_{i}}\right)^{1 / n}}{M} \psi_{1}(r)+\psi_{2}(r)
\end{aligned}
$$

where

$$
\begin{aligned}
& \psi_{1}(r)=\frac{\psi(r)}{\left\{(F /(G+H))\left[((G / F)+(H / F)) x^{2}-2(H / F) x+(1+(H / F))\right]\right\}^{1 / 2}}, \\
& \psi_{2}(r)=\frac{\sigma_{0}}{\left\{(F /(G+H))\left[((G / F)+(H / F)) x^{2}-2(H / F) x+(1+(H / F))\right]\right\}^{1 / 2}} .
\end{aligned}
$$

The equation of equilibrium for a rotating disc with varying thickness can be written as

$$
\frac{d}{d r}\left(r h \sigma_{r}\right)-h \sigma_{\theta}+\rho \omega^{2} r^{2} h=0
$$

Integrating (2.21) within limits $a$ to $b$ and using (2.1) and (2.2)

$$
\sigma_{\theta_{\text {avg }}}=\frac{1}{A_{0}} \rho \omega^{2} I_{0}
$$

Substituting $\sigma_{\theta}$ from (2.18) into (2.2)

$$
\frac{\left(\dot{u}_{r_{i}}\right)^{1 / n}}{M}=\frac{A_{0} \sigma_{\theta_{\text {avg }}}-\int_{a}^{b} \psi_{2}(r) \cdot h d r}{\int_{a}^{b} \psi_{1}(r) \cdot h d r} .
$$

Using (2.22) and (2.23), (2.18) becomes

$$
\sigma_{\theta}=\frac{\psi_{1}(r)\left[\rho \omega^{2} I_{0}-\int_{a}^{b} \psi_{2}(r) \cdot h d r\right]}{\int_{a}^{b} \psi_{1}(r) \cdot h d r}+\psi_{2}(r)
$$


Integrating (2.21) within limits $a$ to $r$ and using (2.1)

$$
\sigma_{r}=\frac{1}{r \cdot h}\left[\int_{a}^{r} \sigma_{\theta} \cdot h d r-\rho \omega^{2} I\right]
$$

Thus the tangential stress $\sigma_{\theta}$ and radial stress $\sigma_{r}$ are determined by (2.24) and (2.25). Then strain rates $\dot{\varepsilon}_{r}, \dot{\varepsilon}_{\theta}$ and $\dot{\varepsilon}_{z}$ are calculated from (2.10), (2.11), and (2.12).

\section{Solution Procedure}

The stress distribution is evaluated from the previous analysis by iterative numerical scheme of computation (Figure 1). In the first iteration, it is assumed that $\sigma_{\theta}=\sigma_{\theta_{\text {avg }}}$ over the entire disc radii. Substituting $\sigma_{\theta_{\text {avg }}}$ for $\sigma_{\theta}$ in (2.25) the first approximation value of $\sigma_{r}$, that is, $\left[\sigma_{r}\right]_{1}$, is obtained. The first approximation of stress ratio, that is, $[x]_{1}$, is obtained by dividing $\left[\sigma_{r}\right]_{1}$ by $\sigma_{\theta}$ which can be substituted in (2.13) to calculate first approximation of $\phi(r)$, that is, $[\phi(r)]_{1}$. Now one carries out the numerical integration of $[\phi(r)]_{1}$ from limits of $a$ to $r$ and uses this value in (2.17) to obtain first approximation of $\psi(r)$, that is, $[\psi(r)]_{1}$. Using this $[\psi(r)]_{1}$ and $\sigma_{0}$ in (2.19) and (2.20), respectively, $\left[\psi_{1}(r)\right]_{1}$ and $\left[\psi_{2}(r)\right]_{1}$ are found, which are used in (2.18) to find second approximation of $\sigma_{\theta}$, that is, $\left[\sigma_{\theta}\right]_{2}$. Using $\left[\sigma_{\theta}\right]_{2}$ for $\sigma_{\theta}$ in (2.25), second approximation of $\sigma_{r}$, that is, $\left[\sigma_{r}\right]_{2}$, is found and then the second approximation of $x$, that is, $[x]_{2}$, is obtained. The iteration is continued till the process converges and gives the values of stresses at different points of the radius grid.

For rapid convergence 75 percent of the value of $\sigma_{\theta}$ obtained in the current iteration has been mixed with 25 percent of the value of $\sigma_{\theta}$ obtained in the last iteration for use in the next iteration, that is, $\sigma_{\theta \text { next }}=.25 \sigma_{\theta \text { previous }}+.75 \sigma_{\theta \text { current }}$.

The strain rates are then calculated from (2.10), (2.11), and (2.12).

\section{Numerical Computations and Discussions}

For the sake of computation of a rotating disc made of $\mathrm{SiC}_{p}$ reinforced $6061 \mathrm{Al}$ matrix composite (Figure 2), we assume that the disc is subjected to an angular velocity of $15,000 \mathrm{rpm}$ and we choose particle size $P=1.7 \mu \mathrm{m}$, particle content $V=20 \%$, and temperature $T=616 \mathrm{~K}$. For the anisotropic material $G / F=1.34, H / F=1.64$ and isotropic material $G / F=1, H / F=1$, creep parameters have been carried out as reported in Gupta and Singh [19]. The creep behavior of the material is described by threshold stress-based creep law by assuming a stress exponent $(n)$ of 8 . The inner radii $a$ and the outer radii $b$ of all the discs are taken as $31.75 \mathrm{~mm}$ and $152.4 \mathrm{~mm}$, respectively. A computer program based on the analysis presented in this paper has been developed to obtain the steady-state creep response of the composite discs with linearly varying thickness in presence of residual stress and obtained results are compared to the disc without residual stress to analyze the importance of residual stress. For the analysis, the tensile residual stress $\left(\Delta \sigma_{\mathrm{y}}\right)$ is taken as $32 \mathrm{MPa}$, as observed by Singh and Rattan [17]. 


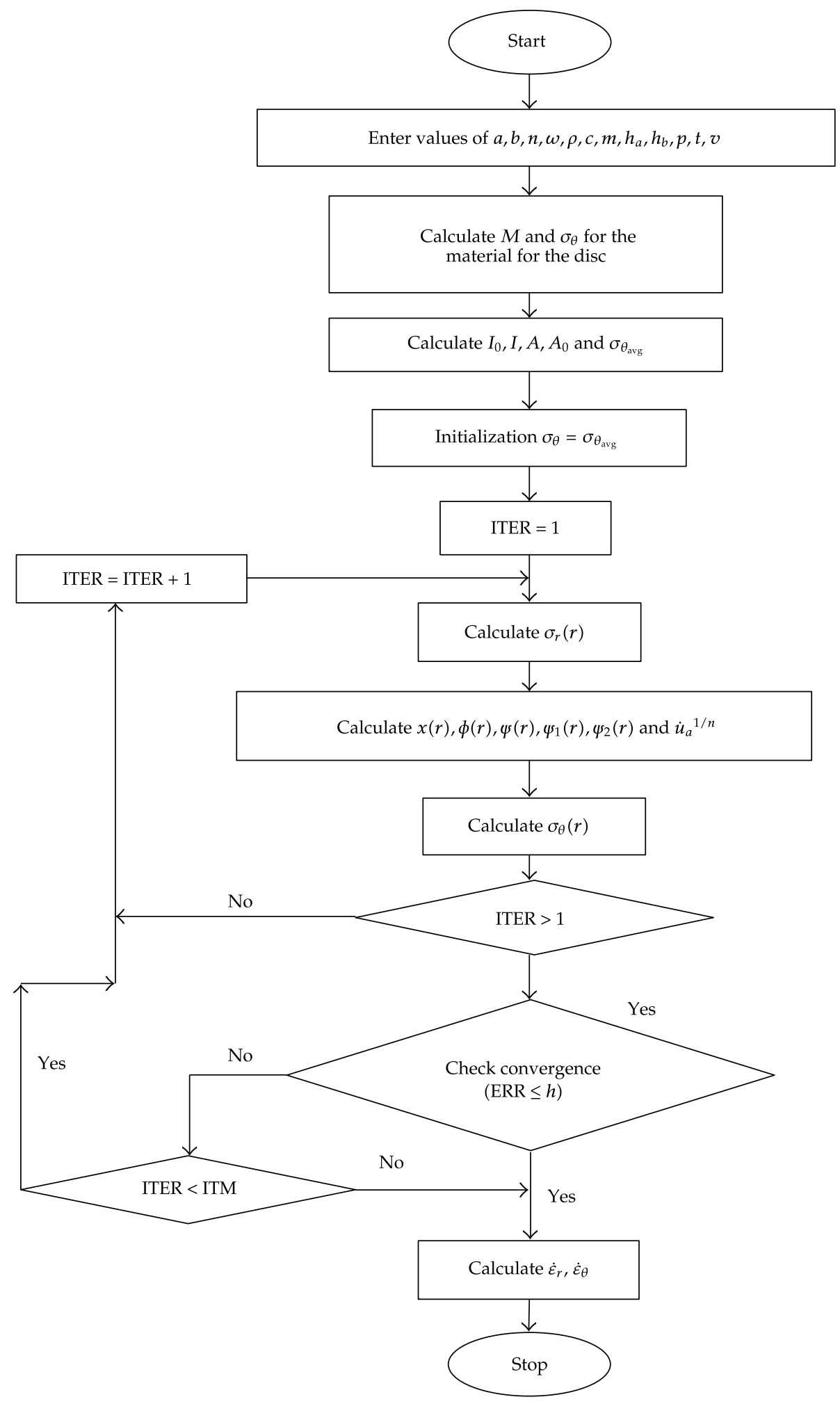

Figure 1: Numerical scheme of computation. 


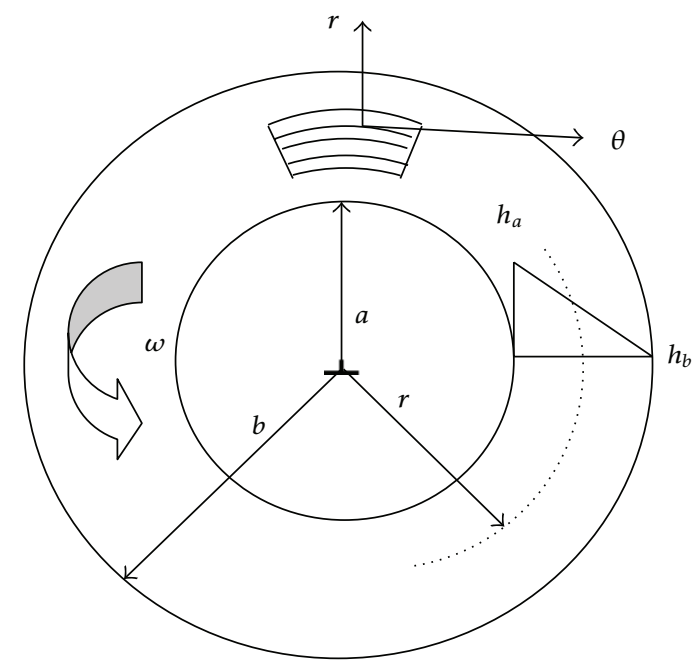

Figure 2: A rotating disc of linearly varying thickness.

For the isotropic/anisotropic discs, linearly varying thickness, that is, $\left(h_{a}=1.44, h_{b}=\right.$ 0.75), has been taken, where $h$ is the thickness of disc ( $\mathrm{mm})$ and the thickness $h$ is assumed to be of the form $h=h_{b}+2 c(b-r)$ and $h_{a}=h_{b}+2 c(b-a)$, where $c=\left(h_{a}-h_{b}\right) / 2(b-a)$ is the slope of a disc.

Using this expression for thickness, (2.1) becomes

$$
\begin{gathered}
A=(r-a)\left[h_{b}+c(2 b-r-a)\right] \\
A_{0}=(b-a)\left[h_{b}+c(b-a)\right] \\
I=\frac{h_{b}}{3}\left(r^{3}-a^{3}\right)+\frac{2 c b}{3}\left(r^{3}-a^{3}\right)-\frac{c}{2}\left(r^{4}-a^{4}\right) \\
I_{0}=\frac{h_{b}}{3}\left(b^{3}-a^{3}\right)+\frac{2 c b}{3}\left(b^{3}-a^{3}\right)-\frac{c}{2}\left(b^{4}-a^{4}\right) .
\end{gathered}
$$

The creep analysis in a rotating disc made of Al-SiC (particle) composite having linearly varying thickness has been carried using isotropic/anisotropic Hoffman yield criterion of yielding and results are compared with those using von Mises yield criterion/Hill's criterion of yielding ignoring difference in yield stresses, that is, $\left(\Delta \sigma_{\mathrm{y}}=0\right)$.

Figure 3 shows the tangential stress in an isotropic/anisotropic rotating disc with linearly varying thickness in presence of residual stress and the results are compared with those for without residual stress. It is concluded that in the isotropic/anisotropic discs, the tangential stress is a little lower in region near the inner radius and slightly higher in region near the outer radius in the presence of residual stress as compared to the disc without residual stress. It is also noted that the effect of residual stress on tangential stress distribution is lesser in an anisotropic rotating disc of linearly varying thickness compared 


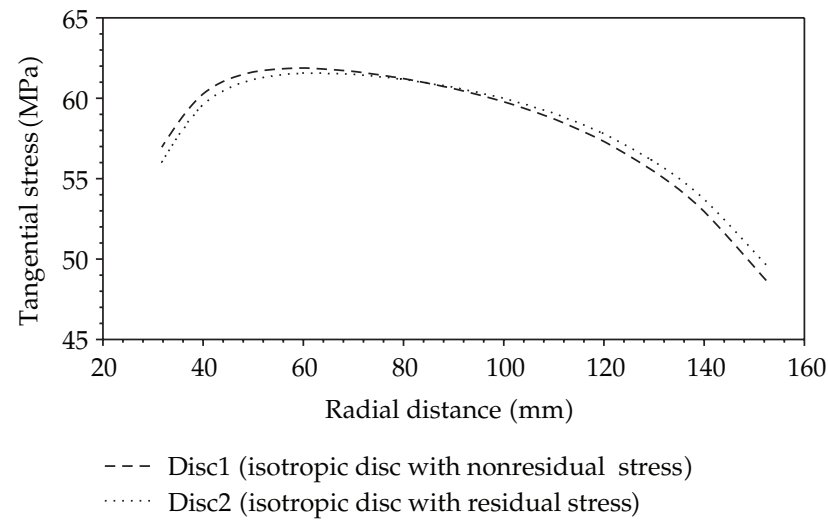

(a)

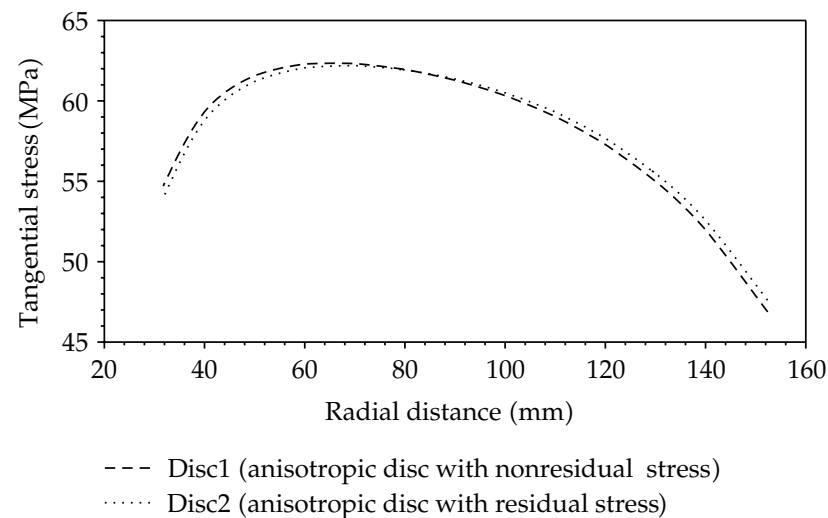

(b)

Figure 3: Variation of tangential stress along the radial distance in the isotropic/anisotropic discs rotating with an angular velocity $15000 \mathrm{rpm}$ at $616 \mathrm{~K}$.

to an isotropic disc of linearly varying thickness. Also the tangential stress becomes more uniform in the presence/absence of residual stress in an anisotropic disc compared to an isotropic disc.

Figure 4 shows the variation of radial stresses along the radius of the isotropic/anisotropic rotating disc in presence/absence of residual stress. It is observed that in presence of residual stress, the radial stress developing due to rotation is slightly lesser than the radial stress of an isotropic/anisotropic disc without residual stress, although the change in the magnitude of radial stress distribution is very small in the isotropic/anisotropic discs with linearly varying thickness due to presence of residual stress. As one moves from the inner radius to the outer radius of the disc, the radial stress increases from zero and reaches a maximum near the middle region of the disc with linearly varying thickness and then starts decreasing towards the outer region.

Figure 5 shows that in presence of tensile residual stress, the tangential strain rates enhance significantly in both the isotropic/anisotropic discs compared to the discs without residual stress. Also the difference in the tangential strain rate caused due to presence and absence of residual stresses (i.e., the residual effect) goes on increasing with radial distance and the extent of increase in difference is maximum in the region near the outer radius in both 


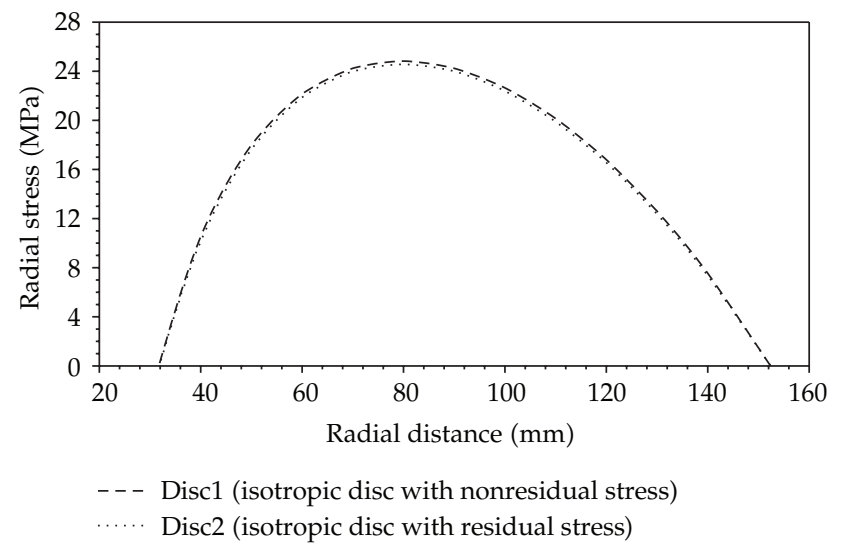

(a)

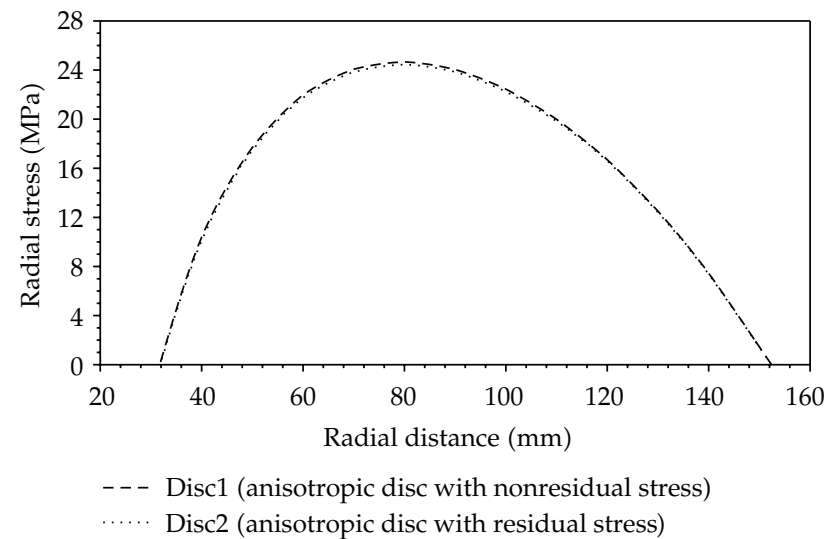

(b)

Figure 4: Variation of radial stress along the radial distance in isotropic/anisotropic discs rotating with an angular velocity $15000 \mathrm{rpm}$ at $616 \mathrm{~K}$.

the isotropic/anisotropic discs having linearly varying thickness. Secondly, it is also noticed that variation in magnitude due to residual stress in an anisotropic disc is smaller compared to that for an isotropic disc. In an isotropic/anisotropic disc with/without residual stress, the tangential strain rates are highest at the inner radius and then decrease continuously, when one moves towards the outer radius of the disc. The trend of variation of tensile strain rate in tangential direction remains the same in an isotropic/anisotropic disc in the presence/absence of residual stress, but the magnitude can be reduced in an anisotropic disc.

Figure 6 shows that in the presence of residual stress, the radial strain rate in an isotropic/anisotropic rotating disc with linearly varying thickness and results are compared with those for without residual stress. By seeing both the discs of isotropic/anisotropic material, it is concluded that in the absence of residual stress, the nature of the radial strain rate which was compressive becomes tensile at the middle of the disc in the presence of residual stress. It is also noticed that the difference in strain rate caused due to the presence and absence of residual stresses is smaller in an anisotropic disc as compared to that for an isotropic disc. Another point to be observed is that the magnitude of radial strain rate firstly 


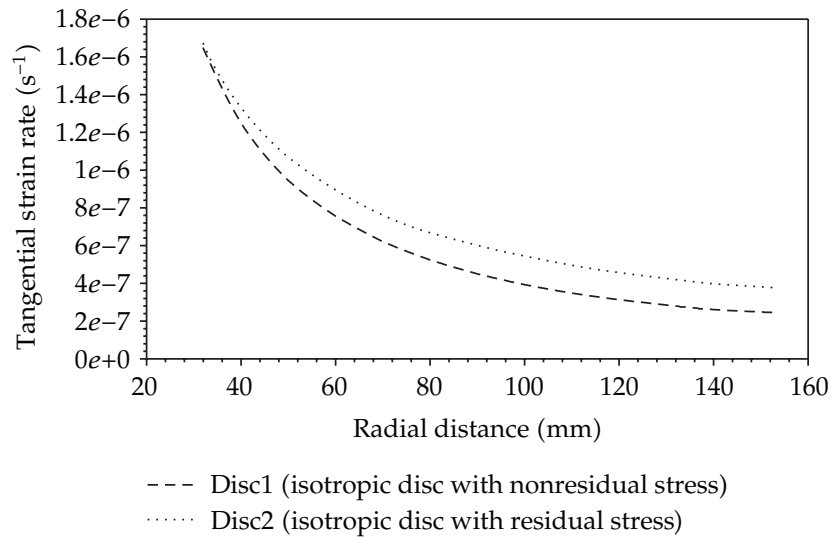

(a)

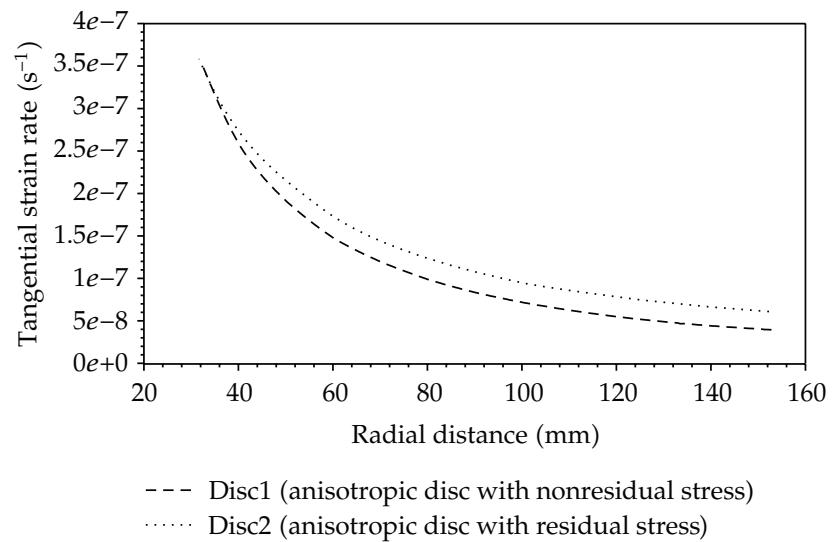

(b)

Figure 5: Variation of tangential strain rate along the radial distance in isotropic/anisotropic discs rotating with an angular velocity $15000 \mathrm{rpm}$ at $616 \mathrm{~K}$.

increases rapidly with radial distance and then starts decreasing. It reaches a minimum before increasing again towards the outer radius in both the isotropic/anisotropic discs with residual/without residual stress.

From previous discussion, it can be concluded that residual stress may cause significant distortion in an isotropic/anisotropic rotating disc having linearly varying thickness, but the magnitude of distortion can be reduced by selecting anisotropic disc.

\section{Conclusion}

The previous results and discussion conclude the following.

(1) The presence of the thermal residual stress developing due to rotation does not significantly affect the distribution of stress in the isotropic/anisotropic disc having linearly varying thickness, but it affects the strain rates significantly.

(2) The magnitude of variation of tangential stress and the radial stress obtained in the anisotropic disc with/without residual stress are relatively smaller as compared to that for isotropic rotating disc. 


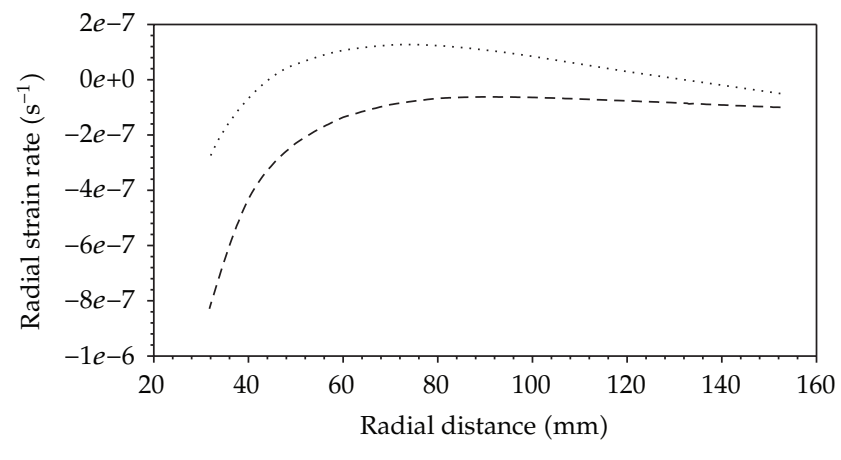

- - - Disc1 (isotropic disc with nonresidual stress)

..... Disc2 (isotropic disc with residual stress)

(a)

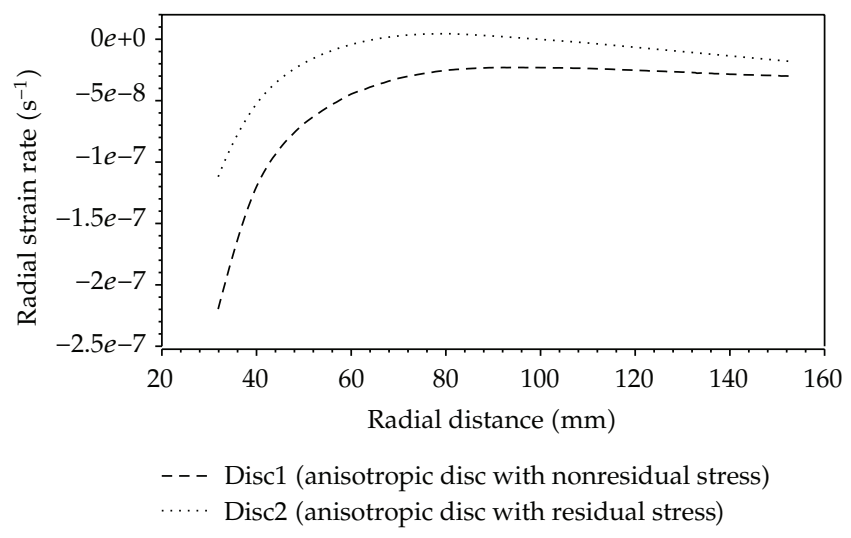

(b)

Figure 6: Variation of radial strain rate along the radial distance in isotropic/anisotropic discs rotating with an angular velocity $15000 \mathrm{rpm}$ at $616 \mathrm{~K}$.

(3) In the isotropic/anisotropic disc, the tangential strain rate is more in presence of thermal residual stress. Also, the extent of difference in creep caused due to the presence/absence of the residual stress increases as one moves towards the outer radius of the disc, although the magnitude of this difference is smaller in anisotropic disc compared to that in isotropic disc.

(4) In the presence of residual stress in the isotropic/anisotropic disc, the nature of radial strain rate changes from compressive to tensile, particularly in the middle region of the disc with linearly varying thickness as compared to the disc without residual stresses. Further, the magnitude of residual's effect in the anisotropic disc is significantly lower as compared to those for the isotropic disc.

(5) For designing a rotating disc with linearly varying thickness operating at elevated temperature, the presence of residual stress needs attention from the point of view of steady-state creep rate. However, the effect of residual stress on the steady-state creep rate in the anisotropic disc is observed to be significantly lower than that observed in the isotropic disc. 


\section{References}

[1] N. S. Bhatnagar, M. P. S. Kulkarni, and V. K. Arya, "Steady-state creep of orthotropic rotating disks of variable thickness," Nuclear Engineering and Design, vol. 91, no. 2, pp. 121-141, 1986.

[2] R. J. Arsenault and M. Taya, "Thermal residual stress in metal matrix composite," Acta Metallurgica, vol. 35, no. 3, pp. 651-659, 1987.

[3] R. S. Mishra and A. B. Pandey, "Some observations on the high-temperature creep behavior of 6061 Al-SiC composites," Metallurgical Transactions A, vol. 21, no. 7, pp. 2089-2090, 1990.

[4] A. B. Pandey, R. S. Mishra, and Y. R. Mahajan, "Steady state creep behaviour of silicon carbide particulate reinforced aluminium composites," Acta Metallurgica Et Materialia, vol. 40, no. 8, pp. 2045 2052, 1992.

[5] L. C. Davis and J. E. Allison, "Residual stresses and their effects on deformation," Metallurgical Transactions A, vol. 24, no. 11, pp. 2487-2496, 1993.

[6] G. K. Hu and G. L. Huang, "Influence of residual stress on the elastic-plastic deformation of composites with two- or three-dimensional randomly oriented inclusions," Acta Mechanica, vol. 141, no. 3, pp. 193-200, 2000.

[7] H. Jahed and R. Shirazi, "Loading and unloading behaviour of a thermoplastic disc," International Journal of Pressure Vessels and Piping, vol. 78, no. 9, pp. 637-645, 2001.

[8] Y. Orcan and A. N. Eraslan, "Elastic-plastic stresses in linearly hardening rotating solid disks of variable thickness," Mechanics Research Communications, vol. 29, no. 4, pp. 269-281, 2002.

[9] S. B. Singh and S. Ray, "Newly proposed yield criterion for residual stress and steady state creep in an anisotropic composite rotating disc," Journal of Materials Processing Technology, vol. 143-144, no. 1, pp. 623-628, 2003.

[10] S. B. Singh and S. Ray, "Modeling the creep in an isotropic rotating disc of Al-SiCw composite in presence of thermal residual stress," in Proceedings of the 3rd International Conference on Advanced Manufacturing Technology (ICAMT '04), pp. 766-770, Kuala Lumpur, Malaysia, May 2004.

[11] V. K. Gupta, S. B. Singh, H. N. Chandrawat, and S. Ray, "Steady state creep and material parameters in a rotating disc of Al-SiCP composite," European Journal of Mechanics, A/Solids, vol. 23, no. 2, pp. 335-344, 2004.

[12] H. Jahed, B. Farshi, and J. Bidabadi, "Minimum weight design of inhomogeneous rotating discs," International Journal of Pressure Vessels and Piping, vol. 82, no. 1, pp. 35-41, 2005.

[13] O. Sayman, "Stress analysis of a thermoplastic composite disc under uniform temperature distribution," Journal of Thermoplastic Composite Materials, vol. 19, no. 1, pp. 61-77, 2006.

[14] S. B. Singh, "One parameter model for creep in a whisker reinforced anisotropic rotating disc of AlSiCw composite," European Journal of Mechanics, A/Solids, vol. 27, no. 4, pp. 680-690, 2008.

[15] F. Z. Xuan, J. J. Chen, Z. Wang, and S. T. Tu, "Time-dependent deformation and fracture of multimaterial systems at high temperature," International Journal of Pressure Vessels and Piping, vol. 86, no. 9, pp. 604-615, 2009.

[16] N. Chamoli, M. Rattan, and S. B. Singh, "Effect of anisotropy on the creep of a rotating disc of Al-SiCp composite," International Journal of Contemporary Mathematical Sciences, vol. 5, no. 11, pp. 509-516, 2010.

[17] S. B. Singh and M. Rattan, "Creep analysis of an isotropic rotating Al-SiCp composite disc taking into account the phase-specific thermal residual stress," Journal of Thermoplastic Composite Materials, vol. 23, no. 3, pp. 299-312, 2010.

[18] J. J. Chen, K. B. Yoon, and S. T. Tu, "Creep behavior of pressurized tank composed of functionally graded materials," Journal of Pressure Vessel Technology, vol. 133, no. 5, Article ID 051401, 2011.

[19] V. Gupta and S. B. Singh, "Modeling anisotropy and steady state creep in a rotating disc of Al-SiCp having varying thickness," International Journal of Scientific E Engineering Research, vol. 2, no. 10, pp. $1-12,2011$.

[20] O. D. Sherby, R. H. Klundt, and A. K. Miller, "Flow stress, subgrain size, and subgrain stability at elevated temperature," Metallurgical Transactions A, vol. 8, no. 6, pp. 843-850, 1977. 


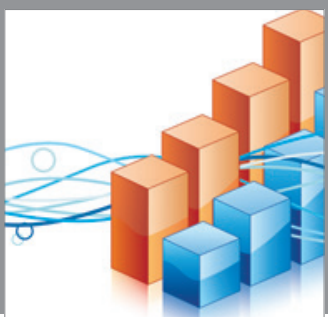

Advances in

Operations Research

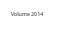

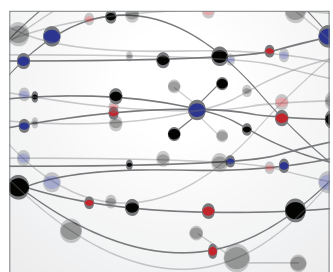

\section{The Scientific} World Journal
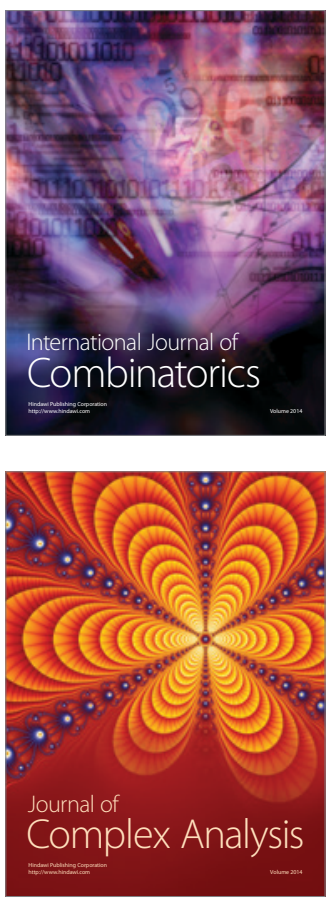

International Journal of

Mathematics and

Mathematical

Sciences
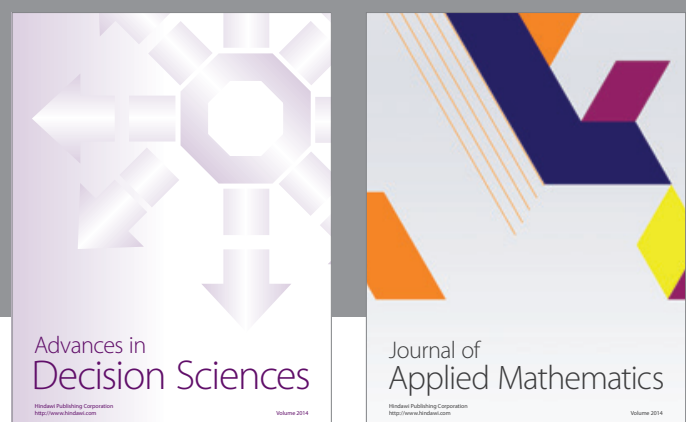

Journal of

Applied Mathematics
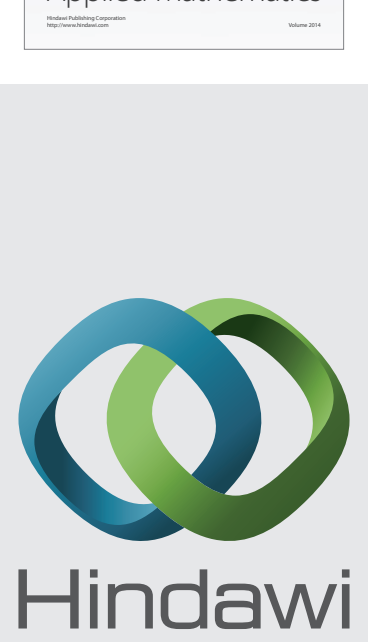

Submit your manuscripts at http://www.hindawi.com
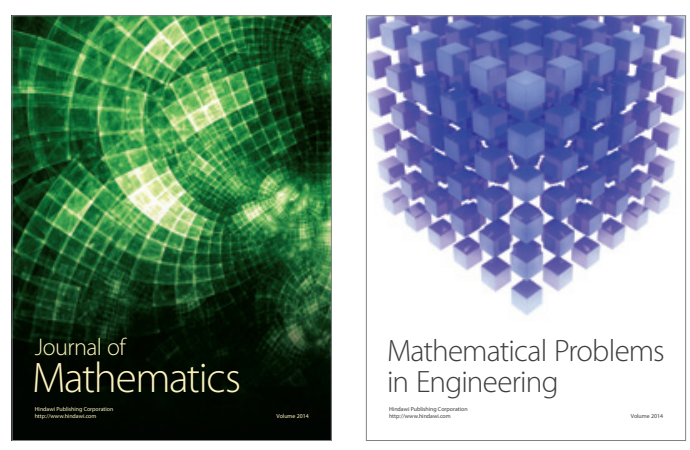

Mathematical Problems in Engineering
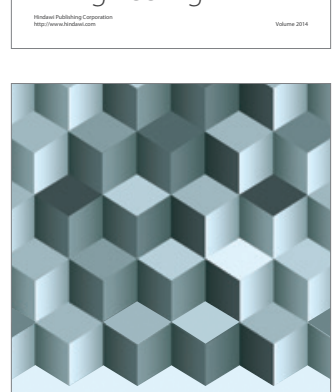

Journal of

Function Spaces
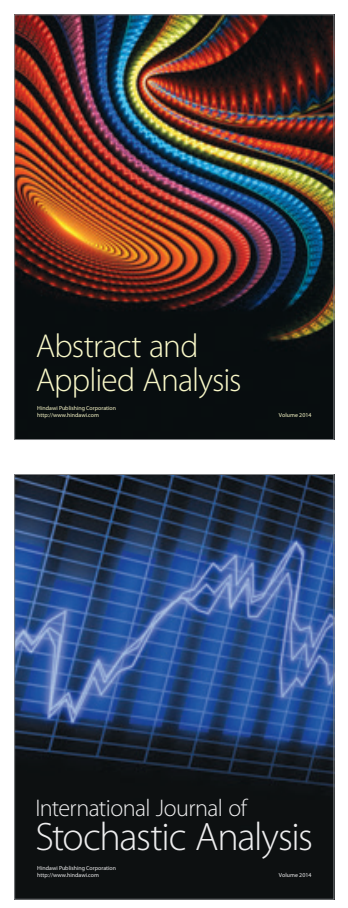

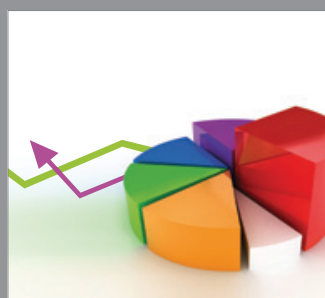

ournal of

Probability and Statistics

Promensencen
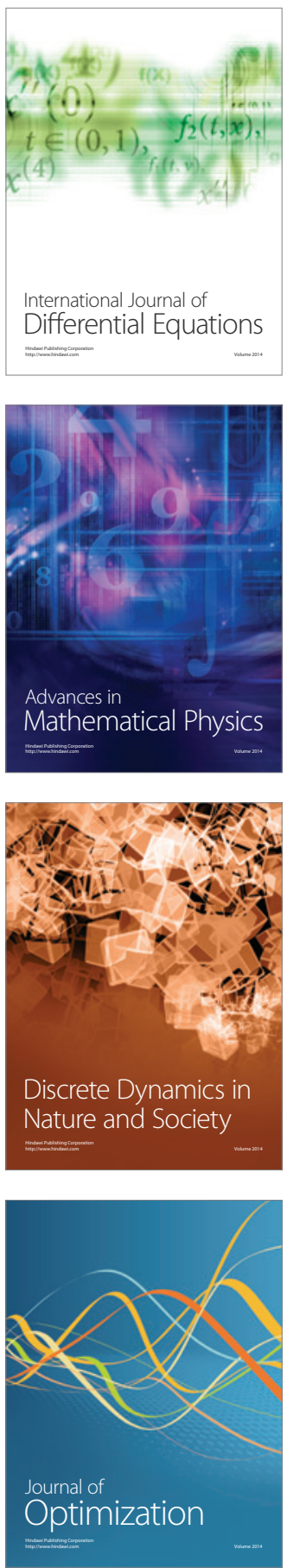